\title{
TOMADA DE DECISÃO NO TRATAMENTO DE EMERGÊNCIAS
}

\section{DECISION TAKING IN THE EMERGENCY SERVICES TREATMENT}

\author{
Samuel Potma Garcias Gonçalves \\ Capitão do Corpo de Bombeiros do Paraná e Engenheiro de Segurança do Trabalho \\ Mestrando no Programa de Pós-Graduação em Engenharia de Produção da UTFPR- \\ Universidade Tecnológica Federal do Paraná - Campus Ponta Grossa \\ Rua Visconde de Bom Retiro, 970, Uvaranas, 84030-080, Ponta Grossa, PR
}

42-3226-5200 potma@,creapr.org.br

\section{Antônio Augusto de Paula Xavier}

Professor do Programa de Pós-Graduação em Engenharia de Produção da UTFPR Universidade Tecnológica Federal do Paraná - Campus Ponta Grossa

Av. Monteiro Lobato, s/n, - Km 4 - 84016-210 - Ponta Grossa- PR

(42) 3220 - 4805 - augustopx@,brturbo.com

\section{Luiz Alberto Pilatti}

Professor do Programa de Pós-Graduação em Engenharia de Produção da UTFPR Universidade Tecnológica Federal do Paraná - Campus Ponta Grossa Av. Monteiro Lobato, s/n, - Km 4 - 84016-210 - Ponta Grossa- PR

(42) 3220 - 4805 - lapilatti@pg.cefetpr.br

\section{RESUMO}

Este artigo apresenta os resultados de uma pesquisa realizada em 2004 e trata do desempenho humano quando exposto a tensão ocasionada pela complexidade, isto é, quando sujeito a um desequilíbrio entre o grau de exigências do trabalho e os recursos disponíveis para gerenciálo; quando o processo de tomada de decisão deve ser muito rápido, em que o tempo de avaliação é pequeno e as conseqüências de cada ato podem ser muito importantes. O objetivo é conhecer a influência, na tomada de decisão em vários momentos da emergência, de fatores como: recursos disponíveis, experiência da pessoa responsável pela tomada de decisão/comandante, tempo para agir, presença de curiosos e estranhos no local e participação anterior do comandante em exercícios simulados. A pesquisa é qualitativa. A coleta de dados foi baseada em um questionário e foi realizada no Corpo de Bombeiros do Paraná, na cidade de Ponta Grossa, com uma amostra de oito oficiais. O resultado obtido na pesquisa mostra 
que quando a tensão, devido à complexidade da emergência cresce, também cresce o grau de influência, para os fatores analisados, no desempenho do comandante.

Palavras chave: Emergência, Estresse, Psicologia do Trabalho, Segurança do Trabalho, Tomada de decisão.

\begin{abstract}
This article presents the results of a research carried through in 2004 and deals with the human performance when exposed to high mental workload, that is, when subject to unbalance between the degree of work requirements and the available resources to manage it; when a decision taking process must be very fast, in wich the evaluation time is small and the consequences due to each act can be very important. The purpose is to know the influence in the decision taking at the very moment of emergency due to factors as: available resources, experience manager, time to act, presence of outsiders and performing of simulated exercises. The research is qualitative. The data collection was based on a quest and carried through in the Fire Department of Paraná state, in the city of Ponta Grossa, with a sample of 8 (eight) officers. The results obtained in the research show that when the mental workload due to the emergency complexity increases, it also increases the influence degree, from the factors analysed, in the performance of the commander.
\end{abstract}

Key Words: Decision taking; Emergency, Safety work, Stress, Work Psycology

\title{
1. INTRODUÇÃO
}

Existem diversas empresas e órgãos públicos envolvidos no trabalho com emergências. O Corpo de Bombeiros é o órgão público mais envolvido em situações complexas, envolvendo perigo de vida tais como incêndios, salvamentos, resgates e socorrismos.

A revisão teórica sobre emergências indica que ela é caracterizada pela presença de curiosos, vítimas, parentes das vítimas e, normalmente, a presença da imprensa na busca de notícias. A experiência do profissional responsável pela tomada de decisão/comandante conta muito e a participação em exercícios simulados é imprescindível. A quantidade de recursos materiais e de pessoal à disposição do comandante é um fator que define qual medida deverá ser tomada nas situações complexas. 
O objetivo do artigo é conhecer, qualitativamente, a influência dos fatores mais diretamente envolvidos no tratamento de emergências para agregar conhecimento à função do gerente, comandante ou da pessoa responsável pela tomada de decisão nessas decisões.

\section{EMERGÊNCIAS}

Emergências envolvem três conjuntos de ações, separadas pelo instante de tempo em que ocorrem. O primeiro conjunto, chamado prevenção, refere-se a medidas executadas no momento anterior ao fato, com o intuito de evitá-lo. O segundo conjunto, chamado tratamento, refere-se a medidas tomadas para minimizar as conseqüências, no momento imediatamente posterior. O terceiro conjunto, chamado investigação, está relacionado ao trabalho realizado algum tempo depois para determinar as causas.

Segundo Castro (1998), emergência é uma situação crítica; acontecimento perigoso ou fortuito. É um caso de urgência. Para Cardella (1999), emergência é a ocorrência de eventos perigosos, não programados sob condição controlada. A emergência se dá quando os riscos se manifestam em fatos reais.

O Corpo de Bombeiros é o órgão público que tem a responsabilidade institucional de combater incêndios, realizar buscas e salvamentos e atividades de defesa civil, devendo estar preparado para atender a todo tipo de emergências. As figuras 1,2,3 e 4 ilustram algumas emergências que são atendidas pelo Corpo de Bombeiros. 

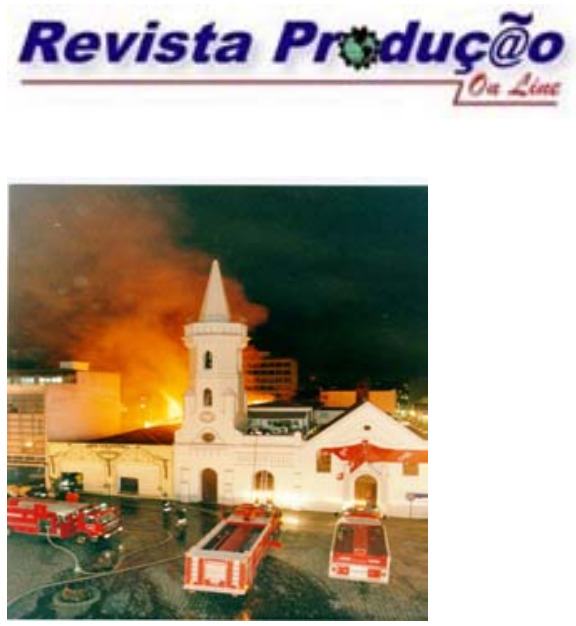

Figura 1: Combate a incêndio Fonte: Corpo de Bombeiros do Paraná, 2005

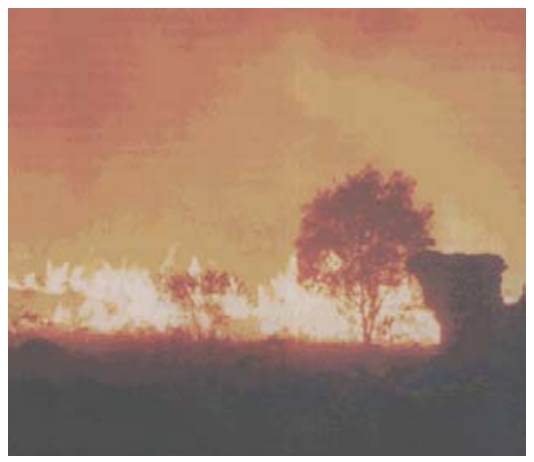

Figura 3: Incêndio em vegetação Fonte: Corpo de Bombeiros do Paraná, 2005

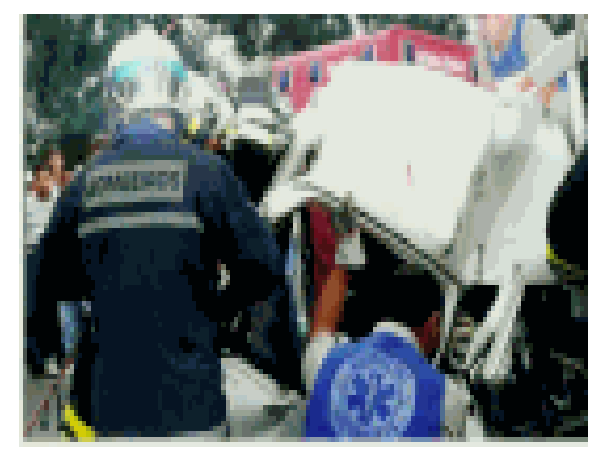

Figura 2: Resgate de vítima de ferragens Fonte: Corpo de Bombeiros do Paraná, 2005

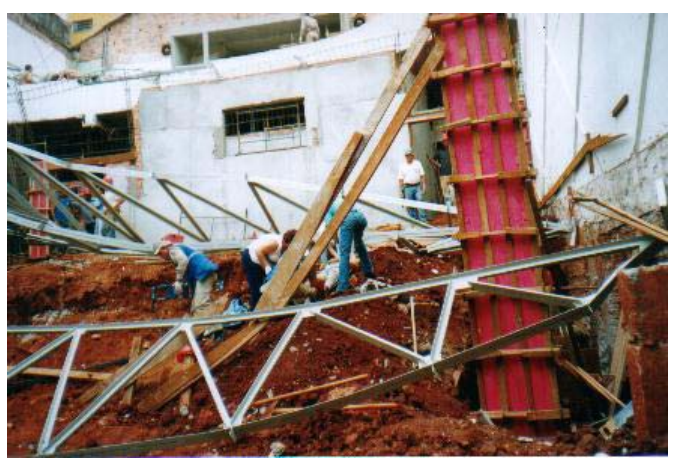

Figura 4: Resgate de vítima de desabamento Fonte: Corpo de Bombeiros do Paraná, 2005

Para Gonçalves e Xavier (2004), as emergências devem ser analisadas previamente para que decisões críticas sejam executadas de modo automático no momento da ocorrência. A pessoa que tem a responsabilidade pela tomada de decisões deve ser adequadamente treinada, pois a possibilidade de cometer falhas em situações de emergências é muito grande. A estratégia é criar exercícios simulados que preparem a organização para o atendimento de emergências. Outros fatores como a presença de terceiros (parentes da vítima, curiosos e a imprensa) interferem no atendimento pela preocupação adicional que causam. Os recursos disponíveis, quanto à qualidade do material e pessoal, que o responsável pela tomada de decisão tenha a sua disposição, na hora da emergência, são imprescindíveis.

O gerente/comandante é avaliado pelo desempenho no seu trabalho e a organização que ele representa é respeitada na medida em que produz bens ou serviços com qualidade. No 
caso de trabalhos envolvendo tensão, ocasionada pela complexidade da tarefa, o desempenho humano apresenta características próprias.

Para Cardela (1999), quando o homem está sob baixas tensões tende a tornar-se descuidado. Incrementos de tensões elevam o desempenho humano até um ponto ótimo. Novos incrementos, a partir deste ponto, provocam queda de desempenho nas situações de emergência, se ele não estiver adequadamente treinado. Os profissionais mais atingidos por esta situação de trabalho são os bombeiros militares, policiais, médicos, enfermeiros, dentre outros. Estes profissionais estão sujeitos a trabalhar no seu dia-a-dia sob tensão acima da tensão ótima variando até a extrema. Esta situação de trabalho sob tensão afeta o desempenho humano em maior ou menor escala, dependendo das características individuais do trabalhador, constituindo um problema a resolver nas organizações.

Outras condições atuam diretamente na performance do comandante/gerente como um processo de seleção eficiente, treinamento e supervisão adequada do profissional. Para Kroemer e Grandjean (2005, p. 166), os cientistas sociais e psicólogos ocupacionais estão de acordo que "o trabalho que considera o potencial e as inclinações da pessoa é levado com interesse, satisfação e boa motivação.” Assim, um trabalhador bem selecionado segundo suas inclinações, com treinamento adequado e que execute o trabalho com satisfação, apresentará melhor desempenho.

No caso das emergências atendidas pelo Corpo de Bombeiros, as características da tarefa e do ambiente exigem protocolos de atendimentos a serem seguidos de forma que o comandante possa detectar e corrigir imediatamente os seus comportamentos inadequados.

\section{TOMADA DE DECISÃO}

O comandante bombeiro militar é um gerente que toma decisões com elevado risco para o que temos de mais valioso - a nossa vida. No seu dia-a-dia, administra a falta de 
efetivo e de recursos financeiros para se equipar e cumprir a sua função de comando. Ele deve ser um líder de pessoas bem treinadas e experientes para resolverem seus problemas.

Segundo Cassaro (2003), o gerente é alguém que é pago para tomar decisões, na maioria das vezes, correndo riscos. Quanto mais elevado estiver na estrutura organizacional, maiores serão as quantidades e o grau de risco.

O profissional que toma decisões deve enxergar seu problema como um todo. É preciso "sair" do problema para visualizá-lo melhor. Assim, deve analisar as variáveis e guiar as decisões de maneira inteligente. Depois que os caminhos estão claros, pode escolher qual caminho seguir.

Uma decisão é uma escolha entre alternativas. Para Drucker (2002), o gerente deve concentrar-se no que é realmente importante, naquilo que é estratégico. Se for de caráter geral, será resolvido por regras gerais. Se não for, deve-se conhecer a respeito da decisão quais os objetivos que aquela decisão pretende atingir, deve-se saber quais as condições limites com maior concisão e clareza possíveis, deve-se sempre começar pelo que é certo e não pelo que é aceitável e a decisão necessita ser discutida por todos. É fundamental que haja discordância para apresentar novas alternativas que não foram pensadas pela pessoa responsável pela tomada de decisão e, finalmente, converter a decisão em ação, definindo quem precisa saber da decisão para concretizá-la.

\section{METODOLOGIA}

Foi feita uma revisão teórica sobre os assuntos emergências e tomada de decisão. Um questionário foi respondido, em 2004, por oito oficiais do Corpo de Bombeiros do Paraná que têm de cinco a quinze anos de serviço e que já passaram e passam, diariamente, por inúmeras situações de risco relacionadas à profissão, durante o plantão operacional. No questionário 
foram criadas três situações hipotéticas em grau crescente de tensão ocasionada pela complexidade: 1) incêndio em residência de madeira com 80 metros quadrados de área, parcialmente tomada pelo fogo (a cozinha, um quarto e uma despensa), sem vítimas, localizada em região próxima ao Corpo de Bombeiros. 2) incêndio em apartamento do segundo pavimento em edificação residencial com quatro pavimentos, dois apartamentos por andar, no horário das quinze horas, localizado em região central, próximo ao Corpo de Bombeiros, com possibilidade da existência de poucas vítimas. 3) incêndio no quinto andar de um edifício comercial com dez pavimentos, sendo totalmente tomado pelo fogo, no horário das quinze horas, localizado em região central, próximo ao Corpo de Bombeiros com a presença de centenas de vítimas no local. Para cada uma destas situações (1, 2 ou 3 ) foram obtidas respostas para a seguinte pergunta: "Qual o grau de importância (influência) dos fatores: terceiros (curiosos e estranhos), recursos disponíveis de material e pessoal, experiência do comandante, tempo para agir e participação anterior em exercícios simulados na tomada de decisão do oficial bombeiro militar no tratamento das emergências? Cite outros que também julgar importantes"; Nestes quesitos, as respostas disponíveis no questionário constavam do nível mais baixo até o mais elevado: pouquíssimo importante, pouco importante, importante, muito importante e extremamente importante.

\section{RESULTADOS}

Os resultados da pesquisa estão apresentados nas tabelas 1,2 e 3, sobre a importância / grau de influência dos fatores de acordo com a complexidade da situação. 
Tabela 1 - Grau de importância dos fatores na situação 1

\begin{tabular}{|l|c|c|c|c|c|}
\hline & $\begin{array}{c}\text { Presença de terceiros } \\
\text { (estranhos, parentes, } \\
\text { imprensa) }\end{array}$ & $\begin{array}{c}\text { Recursos } \\
\text { disponíveis em } \\
\text { material e pessoal }\end{array}$ & $\begin{array}{c}\text { Experiência do } \\
\text { Comandante }\end{array}$ & $\begin{array}{c}\text { Tempo } \\
\text { para agir }\end{array}$ & $\begin{array}{c}\text { Participação anterior do } \\
\text { Comandante em } \\
\text { exercícios simulados }\end{array}$ \\
\hline $\begin{array}{l}\text { Pouquissimo } \\
\text { importante }\end{array}$ & $\mathbf{2}$ & $\mathbf{0}$ & $\mathbf{0}$ & $\mathbf{0}$ & $\mathbf{0}$ \\
\hline $\begin{array}{l}\text { Pouco } \\
\text { importante }\end{array}$ & $\mathbf{3}$ & $\mathbf{0}$ & $\mathbf{0}$ & $\mathbf{1}$ & $\mathbf{3}$ \\
\hline Importante & $\mathbf{3}$ & $\mathbf{5}$ & $\mathbf{7}$ & $\mathbf{2}$ & $\mathbf{4}$ \\
\hline $\begin{array}{l}\text { Muito } \\
\text { Importante }\end{array}$ & $\mathbf{0}$ & $\mathbf{1}$ & $\mathbf{1}$ & $\mathbf{3}$ & $\mathbf{1}$ \\
\hline $\begin{array}{l}\text { Extremamente } \\
\text { Importante }\end{array}$ & $\mathbf{0}$ & $\mathbf{2}$ & $\mathbf{0}$ & $\mathbf{2}$ & $\mathbf{0}$ \\
\hline
\end{tabular}

Fonte: Gonçalves e Xavier (2004, p. 36).

Tabela 2 - Grau de importância dos fatores na situação 2

\begin{tabular}{|l|c|c|c|c|c|}
\hline & $\begin{array}{c}\text { Presença de terceiros } \\
\text { (estranhos, parentes, } \\
\text { imprensa) }\end{array}$ & $\begin{array}{c}\text { Recursos } \\
\text { disponíveis } \\
\text { em material e } \\
\text { pessoal }\end{array}$ & $\begin{array}{c}\text { Experiência do } \\
\text { Comandante }\end{array}$ & $\begin{array}{c}\text { Tempo } \\
\text { para agir }\end{array}$ & $\begin{array}{c}\text { Participação anterior do } \\
\text { Comandante em } \\
\text { exercícios simulados }\end{array}$ \\
\hline $\begin{array}{l}\text { Pouquissimo } \\
\text { importante }\end{array}$ & $\mathbf{0}$ & $\mathbf{0}$ & $\mathbf{0}$ & $\mathbf{0}$ & $\mathbf{0}$ \\
\hline Pouco importante & $\mathbf{3}$ & $\mathbf{0}$ & $\mathbf{0}$ & $\mathbf{0}$ & $\mathbf{0}$ \\
\hline Importante & $\mathbf{5}$ & $\mathbf{0}$ & $\mathbf{1}$ & $\mathbf{0}$ & $\mathbf{3}$ \\
\hline Muito Importante & $\mathbf{0}$ & $\mathbf{3}$ & $\mathbf{6}$ & $\mathbf{1}$ & $\mathbf{3}$ \\
\hline $\begin{array}{l}\text { Extremamente } \\
\text { Importante }\end{array}$ & $\mathbf{0}$ & $\mathbf{5}$ & $\mathbf{1}$ & 7 & 2 \\
\hline
\end{tabular}

Fonte: Gonçalves e Xavier (2004, p. 37).

Tabela 3 - Grau de importância dos fatores na situação 3

\begin{tabular}{|l|c|c|c|c|c|}
\hline & $\begin{array}{c}\text { Presença de terceiros } \\
\text { (estranhos, parentes, } \\
\text { imprensa) }\end{array}$ & $\begin{array}{c}\text { Recursos } \\
\text { disponíveis em } \\
\text { material e pessoal }\end{array}$ & $\begin{array}{c}\text { Experiência do } \\
\text { Comandante }\end{array}$ & $\begin{array}{c}\text { Tempo } \\
\text { para agir }\end{array}$ & $\begin{array}{c}\text { Participação anterior do } \\
\text { Comandante em } \\
\text { exercícios simulados }\end{array}$ \\
\hline $\begin{array}{l}\text { Pouquissimo } \\
\text { importante }\end{array}$ & $\mathbf{0}$ & $\mathbf{0}$ & $\mathbf{0}$ & $\mathbf{0}$ & $\mathbf{0}$ \\
\hline $\begin{array}{l}\text { Pouco } \\
\text { importante }\end{array}$ & $\mathbf{1}$ & $\mathbf{0}$ & $\mathbf{0}$ & $\mathbf{0}$ & $\mathbf{0}$ \\
\hline Importante & $\mathbf{2}$ & $\mathbf{0}$ & $\mathbf{0}$ & $\mathbf{0}$ & $\mathbf{0}$ \\
\hline $\begin{array}{l}\text { Muito } \\
\text { Importante }\end{array}$ & $\mathbf{3}$ & $\mathbf{0}$ & $\mathbf{2}$ & $\mathbf{0}$ & $\mathbf{3}$ \\
\hline $\begin{array}{l}\text { Extremamente } \\
\text { Importante }\end{array}$ & $\mathbf{2}$ & $\mathbf{8}$ & $\mathbf{6}$ & $\mathbf{8}$ & $\mathbf{5}$ \\
\hline
\end{tabular}

Fonte: Gonçalves e Xavier (2004, p. 38). 
Dentre os itens adicionais julgados importantes pelos oficiais pesquisados, constaram as seguintes respostas:

Situação 1) Nada foi constatado;

Situação 2) Comunicações (2 citações), apoio de outros órgãos públicos (2 citações) e conhecimento do local (1 citação);

Situação 3) Comunicações (6 citações), apoio de outros órgãos, como da Polícia Militar e da Companhia de energia elétrica (4 citações), número de vítimas e seu comportamento (2 citações), o preparo físico dos bombeiros (2 citações), confiança no material e nos outros bombeiros (2 citações), recursos disponíveis de terceiros, boas condições de recursos materiais e conhecer previamente o local (1 citação).

\section{DISCUSSÃO DOS RESULTADOS}

A tabela 4 mostra que o fator terceiros, presença de curiosos, estranhos, parentes da vítima e imprensa é o único que diminuiu em importância quando se aumentou a tensão, possivelmente porque, quando se aumenta a tensão em escala extrema, pela complexidade da emergência, outros fatores (tempo para agir, recursos disponíveis em material e pessoal) assumem maior importância. Esses outros fatores passam a requerer toda a atenção do comandante e não sobra "espaço", na mente do comandante, para preocupações com os terceiros (curiosos e estranhos) presentes no local da emergência.

Tabela 4 - Fator presença de terceiros nas 3 situações em grau crescente de complexidade

\begin{tabular}{|l|c|c|c|}
\hline & Situação 1 & Situação 2 & Situação 3 \\
\hline Pouquissimo importante & $\mathbf{2}$ & $\mathbf{0}$ & $\mathbf{0}$ \\
\hline Pouco importante & $\mathbf{3}$ & $\mathbf{3}$ & $\mathbf{1}$ \\
\hline Importante & $\mathbf{3}$ & $\mathbf{5}$ & $\mathbf{2}$ \\
\hline Muito Importante & $\mathbf{0}$ & $\mathbf{0}$ & $\mathbf{3}$ \\
\hline Extremamente Importante & $\mathbf{0}$ & $\mathbf{0}$ & $\mathbf{2}$ \\
\hline
\end{tabular}

Fonte: Gonçalves e Xavier (2004, p. 38). 
A tabela 5 indica que os recursos disponíveis em material e pessoal também são vitais para a tomada de decisão e crescem em importância quando a situação é mais tensa. Nenhuma instituição terá à disposição todos os recursos em todos os lugares no tempo necessário. No entanto, deve-se prever antecipadamente o modo mais rápido de se dispor deles quando necessário.

Tabela 5 - Fator recursos disponíveis nas 3 situações em grau crescente de complexidade

\begin{tabular}{|l|c|c|c|}
\hline & Situação 1 & Situação 2 & Situação 3 \\
\hline Pouquissimo importante & $\mathbf{0}$ & $\mathbf{0}$ & $\mathbf{0}$ \\
\hline Pouco importante & $\mathbf{0}$ & $\mathbf{0}$ & $\mathbf{0}$ \\
\hline Importante & $\mathbf{5}$ & $\mathbf{0}$ & $\mathbf{0}$ \\
\hline Muito Importante & $\mathbf{1}$ & $\mathbf{3}$ & $\mathbf{0}$ \\
\hline Extremamente Importante & $\mathbf{2}$ & $\mathbf{5}$ & $\mathbf{8}$ \\
\hline
\end{tabular}

Fonte: Gonçalves e Xavier (2004, p. 39).

A tabela 6 mostra que o fator experiência (já ter trabalhado, em ocorrências similares, anteriormente, no corpo de bombeiros) é uma defesa à situação de elevada tensão, pois o que impressiona mais aos comandantes novos, aos mais velhos não assusta mais. A pesquisa mostrou que o fator experiência anterior varia de importante a extremamente importante quando a situação modificou da um para a três.

Tabela 6 - $O$ fator experiência do comandante nas 3 situações em grau crescente de complexidade

\begin{tabular}{|l|c|c|c|}
\hline & Situação 1 & Situação 2 & Situação 3 \\
\hline Pouquissimo importante & $\mathbf{0}$ & $\mathbf{0}$ & $\mathbf{0}$ \\
\hline Pouco importante & $\mathbf{0}$ & $\mathbf{0}$ & $\mathbf{0}$ \\
\hline Importante & $\mathbf{7}$ & $\mathbf{1}$ & $\mathbf{0}$ \\
\hline Muito Importante & $\mathbf{1}$ & $\mathbf{6}$ & $\mathbf{2}$ \\
\hline Extremamente Importante & $\mathbf{0}$ & $\mathbf{1}$ & $\mathbf{6}$ \\
\hline
\end{tabular}

Fonte: Gonçalves e Xavier (2004, p. 39).

A tabela 7 indica que o fator tempo para agir, escasso nesta hora, torna-se absolutamente imprescindível. Quando o grau de tensão aumentou, a importância do tempo 
disponível para a ação aumentou proporcionalmente, ou seja, quanto mais este é necessário, mais ele é insuficiente, na hora da emergência.

Tabela 7 - $O$ fator tempo para agir nas 3 situações em grau crescente de complexidade

\begin{tabular}{|l|c|c|c|}
\hline & Situação 1 & Situação 2 & Situação 3 \\
\hline Pouquissimo importante & $\mathbf{0}$ & $\mathbf{0}$ & $\mathbf{0}$ \\
\hline Pouco importante & $\mathbf{1}$ & $\mathbf{0}$ & $\mathbf{0}$ \\
\hline Importante & $\mathbf{2}$ & $\mathbf{0}$ & $\mathbf{0}$ \\
\hline Muito Importante & $\mathbf{3}$ & $\mathbf{1}$ & $\mathbf{0}$ \\
\hline Extremamente Importante & $\mathbf{2}$ & 7 & $\mathbf{8}$ \\
\hline
\end{tabular}

Fonte: Gonçalves e Xavier (2004, p. 40).

A tabela 8 mostra que a participação, anteriormente, do comandante em exercícios simulados utilizados nos treinamentos diários dos bombeiros como uma maneira de antecipar problemas em futuras ocorrências mostra que quanto maior é a gravidade da ocorrência, mais eles são necessários.

Tabela 8 - O fator participação em exercícios simulados nas 3 situações em grau crescente de complexidade

\begin{tabular}{|l|c|c|c|}
\hline & Situação 1 & Situação 2 & Situação 3 \\
\hline Pouquissimo importante & $\mathbf{0}$ & $\mathbf{0}$ & $\mathbf{0}$ \\
\hline Pouco importante & $\mathbf{3}$ & $\mathbf{0}$ & $\mathbf{0}$ \\
\hline Importante & $\mathbf{4}$ & $\mathbf{3}$ & $\mathbf{0}$ \\
\hline Muito Importante & $\mathbf{1}$ & $\mathbf{3}$ & $\mathbf{3}$ \\
\hline Extremamente Importante & $\mathbf{0}$ & $\mathbf{2}$ & $\mathbf{5}$ \\
\hline
\end{tabular}

Fonte: Gonçalves e Xavier (2004, p. 40).

Os outros fatores, acrescidos pelos entrevistados, completam o resultado da pesquisa, como: a necessidade do comandante ter garantidas as comunicações por rádios com os bombeiros que participam das operações e com os que estão no quartel à espera de reforçar o contingente; o apoio de outros órgãos como a Polícia Militar e a companhia de energia elétrica; o número de vítimas e o comportamento delas durante a ocorrência e a confiabilidade 
no material, esclarecendo o que é importante para o comandante do corpo de bombeiros no atendimento às situações de emergências.

\section{CONCLUSÃO}

O assunto tomada de decisão em situações de emergências é um campo aberto para pesquisas, dentro da engenharia de produção. Muitas empresas trabalham com a atividade de emergências, outras podem ser obrigadas a administrar uma ocorrência em seu interior. Todas devem estar preparadas para essas situações.

Alguns fatores como: o tempo para agir, os recursos disponíveis de materiais e de pessoas, a presença de terceiros (como curiosos, parentes das vítimas e até mesmo os órgãos de imprensa), a experiência do comandante, participação em exercícios simulados exercem forte influência sobre quem toma decisões em emergências dependendo da complexidade da situação. Outros fatores são igualmente importantes e devem ser incluídos em futuras pesquisas, a exemplo daqueles obtidos na pesquisa que são: comunicações, o número de vítimas e o comportamento delas durante a ocorrência, a confiabilidade no material e apoio de outros órgãos.

Para a decisão a ser tomada, enquanto que nas situações mais simples, com tensões menores, quando as decisões são menos importantes pelos seus efeitos, os fatores têm peso menor. Nas situações mais complexas, em ordem crescente de tensão, os fatores têm peso maior para o comandante.

Em síntese, quanto maior a tensão devido à complexidade da ocorrência, maior o grau de influência que os fatores exercem sobre o desempenho do comandante, que é a pessoa responsável pela tomada de decisão. 


\section{REFERÊNCIAS}

CARDELA. B. Segurança no trabalho e prevenção de acidentes: uma abordagem holística. São Paulo: Atlas.1999.

CASSARO, A.C. Sistema de informações para tomada de decisões. 3. ed. São Paulo: Pioneira Thomson Learning.2003.

CASTRO, A. L. C. Glossário de defesa civil: Estudos de Riscos e Medicina de Desastres. Brasília: Ministério do Planejamento e Orçamento, Departamento de Defesa Civil.1998.

CORPO DE BOMBEIROS DO PARANÁ: disponível em http://www.pr.gov.br/bombeiros/> Acesso em 10 Maio 2005.

DRUCKER, P. F. O melhor de Peter Drucker: o homem. Trad. Maria Lucia L. Rosa. São Paulo: Nobel.2002

GONÇALVES, S. P. G.; XAVIER, A. A. P. Tomada de decisão em situações de emergência: Monografia, Curso de Especialização em Gestão Industrial - Produção e Manutenção, CEFET-PR.2004.

KROEMER, K. H. E. ; GRANDJEAN, E. Manual de ergonomia: adaptando o trabalho ao homem. 5. ed. Porto Alegre: Bookman. 2005. 